\title{
$\begin{array}{ll}\text { Research Square } & \text { Preprints are preliminary reports that have not undergone peer review. } \\ \text { They should not be considered conclusive, used to inform clinical practice, } & \text { or referenced by the media as validated information. }\end{array}$
}

\section{Astragaloside IV Promotes the Angiogenic Capacity of Adipose-derived Mesenchymal Stem Cells in a Hindlimb Ischemia Model by FAK Phosphorylation via CXCR2}

\section{Weiyi Wang}

Xi'an Jiaotong University Medical College First Affiliated Hospital

\section{Zekun Shen}

Xi'an Jiaotong University Medical College First Affiliated Hospital

\section{Yanan Tang}

Xi'an Jiaotong University Medical College First Affiliated Hospital

\section{Bingyi Chen}

Xi'an Jiaotong University Medical College First Affiliated Hospital Jinxing Chen

Xi'an Jiaotong University Medical College First Affiliated Hospital Jiaxuan Hou

Xi'an Jiaotong University Medical College First Affiliated Hospital

Jiayan Li

Xi'an Jiaotong University Medical College First Affiliated Hospital

\section{Mengzhao Zhang}

Xi'an Jiaotong University Medical College First Affiliated Hospital

\section{Shuang Liu}

Xi'an Jiaotong University Medical College First Affiliated Hospital

\section{Yifan Mei}

Xi'an Jiaotong University Medical College First Affiliated Hospital

\section{Liwei Zhang}

Xi'an Jiaotong University Medical College First Affiliated Hospital

Shaoying Lu ( $\square$ robertlu@mail.xjtu.edu.cn )

Xi'an Jiaotong University Medical College First Affiliated Hospital

\section{Research}

Keywords: Angiogenesis, Mesenchymal stem cells, Astragaloside IV, Ischemic hindlimb, Chemokine 
DOI: https://doi.org/10.21203/rs.3.rs-838346/v1

License: (c) (1) This work is licensed under a Creative Commons Attribution 4.0 International License. Read Full License

Version of Record: A version of this preprint was published at Phytomedicine on December 1st, 2021. See the published version at https://doi.org/10.1016/j.phymed.2021.153908. 


\section{Abstract}

\section{Background}

Therapeutic angiogenesis by transplantation of autologous/allogeneic adipose stem cells (ADSCs) is a potential method for the treatment of severe limb ischemia (CLI). However, the therapeutic efficiency is limited by poor viability, adhesion, migration and differentiation after cell transplantation into the target area. Astragaloside IV (AS-IV), one of the main active components of Astragalus, has been widely used in the treatment of ischemic diseases and can promote cell proliferation and angiogenesis.

\section{Methods}

ADSCs were obtained and pretreated with the different concentration of AS-IV. In vitro, we analyzed the influence of AS-IV on ADSC proliferation, migration, angiogenesis and recruitment of human umbilical vein endothelial cells (HUVECs) and analyzed the relevant molecular mechanism. In vivo, we injected drug-pretreated ADSCs into a Matrigel or hindlimb ischemia model and evaluated the therapeutic effect by the laser Doppler perfusion index, immunofluorescence and histopathology.

\section{Results}

In vitro experiments showed that AS-IV improved ADSC migration, angiogenesis and endothelial recruitment. The molecular mechanism may be related to the upregulation of CXCR2 to promote the phosphorylation of focal adhesion kinase (FAK). In vivo, Matrigel plug assay showed that ADSCs pretreated with AS-IV have stronger angiogenic potential. The laser Doppler perfusion index of the hindlimbs of mice in the ADSC/AS-IV group was significantly higher than the laser Doppler perfusion index of the hindlimbs of mice of the ADSC group and the control group, and the microvessel density was significantly increased.

\section{Conclusion}

AS-IV pretreatment can improve the migration, angiogenesis and endothelial cell recruitment of ADSCs by FAK phosphorylation via CXCR2, as well as the therapeutic effect on ischemic hindlimb model, which will bring new insights into the treatment of severe limb ischemia.

\section{Introduction}

$\mathrm{CLI}$, an ischemic disease that occurs in the extremities, is a serious form of peripheral arterial disease that can cause resting pain, ulcers and tissue necrosis [1]. Patients suffering from CLI always have poor physical function and have a very high risk of amputation. For the treatment of CLI, surgical methods (such as arterial reconstruction and arterial bypass) to increase blood perfusion were usually used in the past. However, $30 \%$ of patients could not receive surgical treatment because of high surgical risk or adverse vascular anatomy [2], and their quality of life was seriously affected. 
In the past 20 years, the using of cytotherapy to rebuild blood vessels and improve limb blood perfusion has become a new treatment for $\mathrm{CLI}$, especially for patients who are not suitable for surgical or interventional surgery. As early as 2002, Tateishi-Yuyama's group transplanted autologous mononuclear bone marrow cells into patients with limb ischemia to achieve therapeutic angiogenesis [3]. Then, various kinds of mesenchymal stem cells (MSCs) were extracted from tissues such as bone marrow, adipose tissue [4], dental pulp [5], skin [6], umbilical cord, and endometrium [7]. MSCs have been postulated and determined to promote angiogenesis through their paracrine effects, differentiation effects (myogenic differentiation and endothelial differentiation), and immunomodulatory effects [8]. Among them, ADSCs are more widely studied and used because they have a stronger cell proliferation rate and angiogenesis effect $[9,10]$ and are easier to extract and autotransplant. The problem that researchers are currently facing is how to improve the therapeutic effect of cell transplantation on the existing basis. In addition to improving the retention of ADSCs in ischemic tissues, improving the migration ability and paracrine function of ADSCs can also produce important benefits [11].

Astragaloside IV, a saponin purified from Astragalus, has been widely used for a long time in traditional Chinese medicine to treat ischemic diseases. Numerous studies have shown that Astragalus has positive effects, such as protecting hypoxic myocardial cells [12], promoting angiogenesis [13], inhibiting muscle damage [14]. The potential mechanisms include activation of the PI3K/Akt signaling pathway, JAK2/STAT3 and ERK1/2 signaling pathways [15], AMPK signaling pathway [16] and overexpression of vascular endothelial growth factor (VEGF) and hypoxia-inducible factor-1a (HIF-1a) [17]. However, there is no report on the effect of AS-IV on ADSCs and its effect on hindlimb ischemia through cell transplantation.

Therefore, in this study, we pretreated ADSCs with AS-IV, analyzed their activity, proliferation, migration and effect on angiogenesis, and tried to find out the molecular mechanism of these effects. Then, we transplanted AS-IV-treated ADSCs into a Matrigel to evaluate its angiogenic potential and into ischemic hindlimb mice to evaluate the therapeutic effect by the laser Doppler perfusion index, histopathology and immunofluorescence.

\section{Methods}

\subsection{Reagents}

Chemical reagents AS-IV (\#HY-N0431), SB225002 (\#HY-16711) and AMD3100 (\#HY-10046) were purchased from MedChemExpress (Monmouth Junction, NJ, USA), and PF573228 (\#T2001) was purchased from TargetMol (Boston, MA, USA). According to the instructions, dry powders of AS-IV, SB225002 and PF573228 were dissolved and diluted with dimethyl sulfoxide (DMSO), and dry powders of AMD3100 were dissolved and diluted with ethanol.

\subsection{Isolation of ADSCs}


Human adipose tissue was obtained from the subcutaneous fat of the upper arm and abdomen of a 30year-old healthy female donor (the First Affiliated Hospital of Xi'an Jiaotong University, China) through liposuction. To extract ADSCs, adipose tissue was cut into small pieces, placed in a centrifuge tube, rinsed with phosphate buffered saline (PBS) and centrifuged (1000 rpm, $5 \mathrm{~min}$ ). The lower liquid was aspirated, the adipose tissue was digested at $37^{\circ} \mathrm{C}$ for 40 minutes using a digestion solution containing $0.2 \%$ collagenase I (\#SCR103, Sigma-Aldrich, St Louis, MO, USA), and the centrifuge tube was shaken several times during digestion. Then, an equal volume of DMEM/F-12 medium containing $10 \%$ fetal bovine serum was added for neutralization, pipetted for 5 minutes and centrifuged (1500 rpm, 10 minutes). After centrifugation, the lowermost cell-containing mixed liquid was aspirated and centrifuged twice (1000 rpm, $5 \mathrm{~min}$ ). Culture medium was added to resuspend the cells, filtered with a 200-mesh cell strainer, and inoculated into a $60 \mathrm{~mm}$ cell culture dish. The medium was changed every other day until the cells reached $80-90 \%$ confluence. The cells can be harvested for expansion and cryopreservation.

\subsection{Cell culture}

HUVECs were purchased from American Type Culture Collection (ATCC, Manassas, VA, USA), and the cells were cultured in Dulbecco's modified Eagle's medium (DMEM) (Gibco, Grand Island, NY, USA) supplemented with $10 \%$ fetal bovine serum (Gemini, Woodland, CA, USA) and 1\% antibiotic-antimycotic (HyClone, Logan, UT, USA). ADSCs were cultured in Dulbecco's modified Eagle's medium F-12 (DMEM/F12) (Gibco) supplemented with $10 \%$ fetal bovine serum and $1 \%$ antibiotic-antimycotic. Cells were incubated at $37^{\circ} \mathrm{C}$ in a $5 \% \mathrm{CO}_{2}$ humidified incubator. ADSCs used in this study were less than 6 passages (P6).

\subsection{Flow cytometry}

Trypsin (HyClone) digested the cells and resuspended with PBS to a concentration of $1 \times 10^{6}$ cells $/ 100 \mu$. The samples were mixed with anti-human CD29-FITC (\#11-0299-41, eBioscience, San Diego, CA, USA), CD44-PE (\#12-0441-81, eBioscience), CD90-FITC (\#11-0909-41, eBioscience), CD105-APC (\#171057-41, eBioscience), CD34 (\#17-0349-41, eBioscience), CD45-PE (\#12-0456-41, eBioscience). Five microliters of antibody solution was added into $100 \mu \mathrm{L}$ PBS with cell suspension and incubated in the dark at $4^{\circ} \mathrm{C}$ for 30 minutes. Finally, a FACSCalibur ${ }^{\text {TM }}$ flow cytometer (BD Biosciences, Franklin Lakes, NJ, USA) was used to analyze the stained cells. FlowJo 10 (BD Biosciences) software was used to analyze the data. The experiment was repeated three times.

\subsection{MTT assay}

ADSCs in logarithmic growth phase were treated with the indicated treatment method, and then the cell viability was detected by 3-(4,5-dimethylthiazole-2-yl) -2,5-diphenyltetrazolium bromide (MTT) (SigmaAldrich). ADSCs were suspended at a concentration of $3 \times 10^{4}$ cells $/ \mathrm{ml}$ and seeded in a 96-well plate at $200 \mu \mathrm{l}$ per well. After incubating for a specific time in the cell culture incubator, add $20 \mu \mathrm{l}$ of MTT was added to $180 \mu \mathrm{l}$ of complete medium per well. After four hours, the liquid was removed from the 96 -well plate, $150 \mu \mathrm{l}$ of DMSO was added, and the plate was shaken for 10 minutes. The absorbance was 
measured at $490 \mathrm{~nm}$ by an ELISA reader (Bio-Rad, Hercules, CA, USA). The experiment was repeated three times.

\subsection{Preparation of conditioned medium}

A total of $5 \times 10^{5}$ cells were inoculated into a $10 \mathrm{~cm}$ cell culture dish and grown in DMEM/F-12 supplemented with $10 \%$ fetal bovine serum and $1 \%$ antibiotic-antimycotic and different concentrations of AS-IV for 48 hours until the cells reached approximately $80 \%$ confluence. Then, the medium was replaced with FBS-free medium and incubated for $48 \mathrm{~h}$. Then, the medium was collected and centrifuged, conditioned medium (CM) was obtained and used for this study. For the inhibitor group, we added an appropriate concentration of inhibitor 1 hour before adding AS-IV, and the subsequent treatment was the same as described above.

\subsection{Wound healing assay}

ADSCs or HUVECs were inoculated in 6-well plates and grown to a $90 \%$ fusion rate. Wounds were generated on the cell monolayer using a $200 \mu$ sterile pipette tip, and the fragments were washed with PBS and replaced with FBS free medium or different groups of CM. The migration of cells to the wound was observed by inverted microscopy at different time points. Under the condition of 100 times magnification, regions were randomly selected in each well, and the cells in each group of 3 wells were quantified in each experiment.

\subsection{Transwell migration assay}

Migration assays were performed in a Boyden chamber (Millipore, Darmstadt, Germany) with a pore size of $8 \mu \mathrm{m}$ to evaluate the migration ability of ADSCs and the recruitment of HUVECs. For migration assays, $1.5 \times 10^{4}$ ADSCs were seeded into a chamber in a 24-well plate, the cells were suspended in $100 \mu$ serumfree medium in the upper chamber, and $600 \mu$ l medium containing 10\% FBS was added to the lower chamber. For recruiting assays, $1.5 \times 10^{4}$ HUVECs were inoculated in the upper chamber, and different groups of CM were added to the lower chamber. After 24 hours, the chamber was washed with PBS 3 times, fixed with $4 \%$ paraformaldehyde for 15 minutes, and stained with $0.1 \%$ crystal violet for 15 minutes. Then, the upper part of the filter was wiped and inverted under a light microscope (200 times magnification) to observe the visible cell count in 3 random visual fields. The experiment was repeated three times.

\section{9 qRT-PCR}

According to the manufacturer's instructions, total RNA was isolated from ADSCs cells using RNAfast 200 reagents (Feijie Biotechnology, Shanghai, China), quantified by absorbance at $260 \mathrm{~nm}$ and reversetranscribed to complementary DNA using a Prime Script RT-PCR kit (Takara Bio, Dalian, China). cDNA was amplified to detect the expression of specific genes using a CFX96 Real-Time PCR system (Bio-Rad) with SYBR-Green PCR Master Mix (Takara Bio). Gene-specific primers were as follows: CXCR2, F: CCTGTCTTACTTTTCCGAAGGAC and R: TTGCTGTATTGTTGCCCATGT; CXCR4, F: ACTACACCGAGGAAATGGGCT and R CCCACAATGCCAGTTAAGAAGA, bFGF, F: 
AGAAGAGCGACCCTCACATCA and R: CGGTTAGCACACACTCCTTTG; VEGF, F:

AGGGCAGAATCATCACGAAGT and R: AGGGTCTCGATTGGATGGCA; GAPDH, F:

GGAGCGAGATCCCTCCAAAAT and R: GGCTGTTGTCATACTTCTCATGG. Gene mRNA expression levels were assessed by the $2^{-\triangle \Delta C t}$ method. GAPDH was used for standardization.

\subsection{Western blot assay}

After treatment under various experimental conditions, ADSCs ware washed 3 times with PBS, and the total protein was separated with RIPA lysis buffer (Beyotime, Shanghai, China) containing protease inhibitor, phosphatase inhibitor and 0.1 M PMSF (Beyotime). Cells were centrifuged at $12,000 \mathrm{~g}$ at $4^{\circ} \mathrm{C}$ for 20 minutes, and cell lysates containing total cell proteins were collected and quantified by the BCA method (Thermo Fisher Scientific, Waltham, MA, USA). The processed protein samples were subjected to SDS-polyacrylamide gel electrophoresis (8-12\%), and then transferred to a polyvinylidene fluoride (PVDF) membrane. After sealing with $5 \%$ skim milk at room temperature for 1 hour to block the nonspecific binding sites, the membrane was incubated with the primary antibody (diluted 1:1000) at $4^{\circ} \mathrm{C}$ overnight. After washing with TBST 3 times, the membrane and peroxidase-coupled secondary antibody were incubated at room temperature for 1 hour. Finally, protein expression was detected by an ECL chemiluminescence detection system (Bio-Rad). The antibodies used in the experiment were as follows: CXCR2(\#ab225732), CXCR4(\#ab181020), p-PXN(Y118)(\#ab109547),t-PXN(\#ab32084) were purchased from Abcam (Cambridge, United Kingdom) [p-FAK(Tyr397)(\#8556), t-FAK(\#3285) and $\beta$-Catenin(\#8480) were purchased from Cell Signaling Technology (Boston, MA, USA).

\subsection{Matrigel tube formation assay}

Fifty microliters of basement membrane matrix gel (Matrigel) (BD Biosciences) was spread on the bottom of a 96 -well plate at $4^{\circ} \mathrm{C}$, and placed at $37^{\circ} \mathrm{C}$ until Matrigel solidified. HUVECs were suspended at a concentration of $1.5 \times 10^{4}$ cells per $150 \mu \mathrm{L}$ in each kind of CM and inoculated onto a previous Matrigelcoated 96-well plate. After 4 hours of incubation, the field of view was randomly photographed, and the number of lumens formed by each sample and the length of the tube were measured with ImageJ software (National Institutes of Health, Bethesda, MD, USA). The experiment was repeated three times.

\subsection{Matrigel plug in vivo assay}

All male C57BL/6 mice used in this study were approved by the ethics committee of the First Affiliated Hospital of Xi'an Jiaotong University, China. To assess the in vivo angiogenic potential of cells, Matrigel plug assays were performed as described in previous literature [18]. Briefly, 6- to 8-week-old C57BL/6 mice were anesthetized with isoflurane, $5 \times 10^{4}$ ADSCs were suspended in $50 \mu \mathrm{L}$ PBS and mixed with $450 \mu \mathrm{L}$ Matrigel, and the cells were subcutaneously transplanted into mice. After 2 weeks, the mice were euthanized, and Matrigel plugs were collected. Half of each plug was used for section staining, and the 


\subsection{Ischemic hind limb mice model and cell injection}

An ischemic hindlimb module was induced as described in previous literature [19]. Each male C57BL/6 mouse aged 6-8 weeks was anesthetized with isoflurane and its left femoral artery was ligated and resected. A total of $1 \times 10^{6}$ cells were intramuscularly transplanted into the ischemic hindlimb area after surgery $(n=6)$. A laser Doppler perfusion imager (Moor Instrements, Devon, United Kingdom) was used to measure blood perfusion in the hind limbs at 0, 3, 7, 14 and 21 days after the operation. The left-to-right ratio (L/R) was used to indicate the relative blood perfusion rate of each mouse's left hind limb. The mice were euthanized 4 weeks after the operation, and their left gastrocnemius muscle was collected to make paraffin-embedded sections for immunohistochemistry and immunofluorescence analysis.

\subsection{H\&E Staining}

Formalin-fixed Matrigel plugs or gastrocnemius specimens were embedded in paraffin. The slides are prepared from $6 \mu \mathrm{m}$ thick sections. For histological analysis, hematoxylin (Sigma-Aldrich) and eosin (Sigma-Aldrich) staining were applied. The experiment was repeated three times.

\subsection{Immunofluorescence staining}

The slices were dewaxed and rehydrated. Endogenous peroxidase was inactivated with $10 \%$ hydrogen peroxide for 10 minutes. The antigen was recovered by pepsin at $37^{\circ} \mathrm{C}$ for 10 minutes. The sections were blocked in 5\% BSA for 30 minutes and then mixed with 1:500 CD31 antibody (\#ab28364; Abcam) and incubated at room temperature for 1 hour. After washing, the slices were incubated with the secondary antibody for 1 hour at room temperature, and the nuclei were stained with DAPI (Sigma-Aldrich) for 5 minutes and sealed with glycerol. The stained image was captured under a positive fluorescence microscope (Olympus, Tokyo, Japan), and the proportion of CD31 + area in each photo was measured by ImageJ software. The experiment was repeated three times.

\subsection{Statistical analyses}

Statistical analyses were conducted using Student's $t$-test for comparisons of 2 groups, and graphs were drawn by using GraphPad Prism 7.0 (San Diego, CA, USA). The data are expressed as the mean \pm SD of three independent experiments. $\mathrm{P}<0.05$ indicated statistically significant.

\section{Results}




\subsection{Characterization and identification of ADSCs}

Monocytes isolated from healthy human adipose tissue were cultured and passaged in a Petri dish, and the cells were in the form of a fibroblast-like spindle and grew in a whirlpool (Fig. 1A). To further identify ADSCs, flow cytometry analysis was used to detect the expression of cell surface markers. The results showed that the representative markers of mesenchymal stem cells (CD29, CD44, CD90, CD105) were positively expressed, and the surface markers of hematopoietic stem cells CD34 and leukocyte surface markers CD45 were negatively expressed. The isolated cells were confirmed to be adipose-derived mesenchymal stem cells (Fig. 1B).

\subsection{AS-IV enhance ADSCs migration}

Different doses $(20-160 \mu \mathrm{m})$ of AS-IV were used to treat ADSCs (DMSO as a solvent control) for 48 hours, and the MTT assay was used to detect cell proliferation. As shown in Fig. 2B, low concentrations of AS-IV had little effect on the proliferation of ADSCs. In contrast, excessive concentrations of AS-IV (80 $\mu \mathrm{m}$ and $160 \mu \mathrm{m})$ can inhibit the growth of ADSCs. However, the scratch experiment showed that, within the appropriate range, AS-IV promoted the migration of ADSCs in a concentration-dependent manner (Fig. 2C), and the Transwell experiment confirmed this phenomenon (Fig. 2D).

\subsection{AS-IV promotes the recruitment of HUVECs and tube formation}

We extracted the CM of ADSCs treated under different conditions and acted on vascular endothelial cells. Wound healing assays and Transwell assays showed that the CM of ADSCs pretreated with AS-IV promoted the migration of HUVECs (Fig. 3A and B), that is, promoted the recruitment of HUVECs by ADSCs. The tube formation experiment (Fig. 3C) also showed that ADSCs pretreated with $40 \mu \mathrm{M}$ AS-IV had a higher effect on angiogenesis, as reflected in the number of tubes formed and the total length of the tubes.

\subsection{AS-IV mediates the expression of CXCR2 and phosphorylation of FAK in a concentration-dependent manner}

Previous studies have shown that chemokines not only play an important role in cell migration but are also important in arteriogenesis and tissue ischemia repair. Therefore, we used RT-PCR to screen angiogenesis related chemokines and their receptors. The results showed that the expression of CXCR2 at the RNA level and protein level increased in a concentration dependent manner in ADSCs treated with AS-IV (Fig. 4a and b), and the CXCR4 level also increased but was excluded in the follow-up verification (Supplemental Fig. 1). At the same time, FAK and paxillin (PXN) were also activated, the expression of $\beta$ Catenin was increased (Fig. $4 \mathrm{C}$ ), and the mRNA expression of the angiogenesis-related factors bFGF and VEGF was increased (Fig. 4D). 


\subsection{SB225002 inhibits the promotion of ADSC migration and angiogenesis due to high expression of CXCR2}

To verify the effect of highly expressed CXCR2 on ADSCs, we added SB225002, a CXCR2 antagonist, at a concentration of $400 \mathrm{nM} 1$ hour before AS-IV treatment to antagonize CXCR2. The results showed that SB225002 significantly reduced the migration of ADSCs caused by AS-IV (Fig. 5A and B) and recruitment of HUVECs (Fig. 5C and D), and there was also a small inhibitory effect in the group treated with SB225005 alone. Consistent with the migration study, SB225005 also inhibited the angiogenesis of ADSCs caused by AS-IV (Fig. 5E). At the protein level, SB225002 successfully inhibited the phosphorylation of FAK induced by AS-IV treatment and its downstream p-PXN and $\beta$-Catenin expression (Fig. 5F).

\subsection{The inhibition of FAK phosphorylation by PF573228 plays a negative role in the migration and angiogenesis of ADSCs}

To verify the relationship between FAK phosphorylation and CXCR2 expression, we used PF573228 at a concentration of $1 \mu \mathrm{M}$ to inhibit FAK phosphorylation 1 hour before AS-IV treatment. The results also showed that the migration of ADSCs and the recruitment of HUVECs were inhibited compared with the control group. Similarly, in the AS-IV treatment group, the inhibitor also reduced its promotion of ADSC migration (Fig. 6A and B) and the recruitment of HUVECs (Fig. 6C and D). This phenomenon was also demonstrated in the tubule formation experiment (Fig. 6E). In subsequent experiments, we analyzed the effect of PF573228 on the protein level, and the results showed that PF573228 did not affect the increase in CXCR2 expression induced by AS-IV, further showing that FAK is activated downstream of CXCR2. The expression of $p$-PXN and $\beta$-Catenin was downregulated as FAK phosphorylation was also inhibited (Fig. $6 \mathrm{~F})$.

\subsection{AS-IV-pretreated ADSCs demonstrate vasculogenic potential in vivo}

Subsequently, we used a Matrigel plug assay to demonstrate the in vivo angiogenesis potential of ADSCs after AS-IV pretreatment. ADSCs and ADSCs pretreated with $40 \mu \mathrm{M}$ AS-IV were mixed with $450 \mu \mathrm{L}$ Matrigel at a concentration of $5 \times 10^{5}$ cells $/ 50 \mu \mathrm{L}$ PBS ( $50 \mu \mathrm{L}$ PBS mixed with $450 \mu \mathrm{L}$ Matrigel as a negative control group) and injected into C57BL/ 6 mice subcutaneously with a syringe. After 2 weeks, the Matrigel plug was recovered and the relative content of hemoglobin was measured using a Drabkin kit. The results showed that ADSCs pretreated with AS-IV formed more functional blood vessels in Matrigel (Fig. 7A) and had higher hemoglobin content (Fig. 7B). To confirm that ADSCs demonstrate vasculogenic potential in Matrigel plugs, we sliced and stained Matrigel plugs. H\&E staining showed that the Matrigel injected with PBS did not contain typical microvascular-like structures, the formation of microvascular- 
like structures occurred in the ADSC group, and the ADSC/AS-IV group showed numerous microvascularlike structures (Fig. 7C). Immunofluorescence staining also reflected the increase in the positive area of CD31 in the ADSC/AS-IV group (Fig. 7D).

\subsection{AS-IV pretreated ADSCs demonstrate therapeutic effects on hind limb ischemia}

We further explored the therapeutic effects of ADSCs in a mouse hindlimb ischemia model. After ligation and resection of the left femoral artery for 24 hours, $1 \subseteq 10^{6}$ cells or PBS was injected into the ischemic hindlimb area, and limb blood perfusion was detected by the laser Doppler perfusion index on Days 0,3 , 7, 14 and 21. The results showed that the blood perfusion rate of the limbs injected with ADSCs/AS-IV was higher than the blood perfusion rate of the limbs injected with ADSCs from Day 7 (Fig. 8A and B). After 28 days, we separated the muscle tissue on the ischemic side, sliced it for immunofluorescence staining, and found that the vascular endothelial cell marker CD31 in the muscles of the ADSC/AS-IV group was significantly increased (Fig. $8 \mathrm{C}$ and D). We also generated the same model and separated the ischemic muscle specimens after 14 days of cytotherapy for H\&E staining to observe the inflammatory response and muscle necrosis in the early stage of ischemic recovery. The results showed that the PBS group had severe muscle damage, accompanied by obvious inflammation infiltration and fibrosis, and the intermuscular space was blurred, while inflammation and muscle degeneration were reduced in the ADSC group, and the reduction was more significant in the ADSC/AS-IV group (Fig. 8E).

\section{Discussion}

$\mathrm{CLI}$ is a serious form of peripheral arterial disease. Recently, cytotherapy has become one of the effective treatment methods for CLI patients who are not suitable for surgical treatment. ADSCs, a kind of MSCs, are widely studied and used in the field of tissue repair because they are easy to obtain and have fewer ethical problems $[20,21]$. However, the therapeutic effect of ADSCs is still limited, because of the weak biological activity or poor migration ability of cells[22], the injected ADSCs will be consumed in large quantities due to the ischemic and hypoxic environment at the injection site. Therefore, increasing the activity of ADSCs is an important method to improve the success rate of cytotherapy.

Astragaloside IV, a natural plant extract, has been shown to have cardiovascular protective effects [23] and can reduce endothelial dysfunction related to diabetes and its complications. In a study of its effect on HUVECs, AS-IV was found to be able to promote endothelial cell proliferation and reduce hypoxia damage $[12,15]$. In this study, we treated ADSCs with AS-IV and expected ADSCs with AS-IV to play a role in promoting biological activity. In vitro, we found that although AS-IV could not promote the proliferation of ADSCs, it could improve their migration ability, HUVEC recruitment and angiogenesis ability. Previous research indicated that the homing ability of ADSCs helps them navigate to the damage site, and migration and surface molecule-mediated adhesion are the core mechanisms of the homing process [24]. That is, AS-IV can enhance the therapeutic effect by promoting the homing ability of ADSCs. At the same time, endothelial cells migration ability and remodeling into tubular structures are also crucial for 
angiogenesis. In this process, many different cytokines are involved in regulating the migration of chemotactic endothelial cells in angiogenesis, such as VEGF, bFGF and angiopoietin [25]. Due to the effect of AS-IV, the ability of ADSCs' to recruit endothelial cells is also greatly increased, and the vascularization ability is also enhanced accordingly.

In view of the role of AS-IV in ADSCs, we focused our attention on the chemokine system, which is closely related to cell movement and recruitment. Chemokines and their receptors have been found to play an important role in tissue ischemia repair, not only in the recruitment of macrophages and endothelial cells, but also in enhancing the proliferation of myoblasts. The major groups of chemokines and their receptors related to angiogenesis are CCRL2/CCR2, CXCL1, CXCL2, CXCL3, CXCL5, CXCL6, CXCL8/CXCR2 and CXCL12/CXCR4 [26]. We screened these chemokines and their receptors by qRT-PCR and found that after AS-IV treatment, the expression of CXCR2 and CXCR4 in ADSCs increased significantly with the concentration gradient. After using their receptor antagonists (Fig. 7 and Supplementary Fig. 1), we found that although many previous studies have verified the positive role of CXCR4 in stem cell migration and the therapeutic effect [27], in this study, CXCR2, but not CXCR4, played a key role. CXCR2 is an important chemokine receptor that mediates cell chemotaxis. CXCR2 is related to inflammatory diseases and immune responses. The upregulation of CXCR2 is also related to tumor angiogenesis and tumor metastasi and can trigger PI3K/Akt, MAPK and other signaling cascades [28]. In this experiment, we found that the overexpression of CXCR2 led to the activation of FAK/PXN and the upregulation of $\beta$ Catenin. Many studies have shown that FAK can be used as an upstream regulator to regulate the expression of $\beta$-Catenin [29], and it can also promote cell migration by forming FAK/Src complexes, activating $\mathrm{PI} 3 \mathrm{~K}$, and regulating the Rho subfamily. At the same time, FAK kinase activity and its autophosphorylation at Y397 are also considered necessary for normal vascular development [30]. PXN, as a cytoskeletal protein located in focal adhesion and related to cell adhesion, is phosphorylated by FAK at its tyrosine residue at position 118 to participate in cell adhesion and metastasis. Studies have confirmed that under the control of the Tie2 promoter and enhancer, transgenic expression of ectopic FAK in endothelial cells increases angiogenesis in hindlimb ischemia and wound-induced angiogenesis models [31]. Meng $F$ et al. also found that after aquaporin 1 overexpression, FAK and $\beta$-Catenin ware upregulated, which improved the endothelial adhesion characteristics of MSCs and then enhanced migration [32], which was also consistent with our findings. To verify the effect of FAK activation on angiogenesis, we used the FAK phosphorylation inhibitor PF573228 to pretreat ADSCs and found that after using PF573228, the migration and angiogenesis of ADSCs promoted by AS-IV was inhibited, the expression of $\mathrm{p}-\mathrm{PXN}$ and $\beta$-Catenin was reduced, and the expression of CXCR2 was not affected, further indicating that CXCR2, as an upstream factor, promotes the activation of FAK and then affects the migration and angiogenesis of ADSCs.

In vivo experiments further verified the angiogenic capacity of ADSCs pretreated with AS-IV. The Matrigel plug assay showed that the ADSC/AS-IV group resulted in better formation of the vascular network and more hemoglobin penetration. The immunofluorescence experiment also showed more CD31 + vascular endothelium, which fully reflected its angiogenesis potential in vivo. We evaluated the therapeutic effect of AS-IV pretreated ADSCs in vivo by using an ischemic hindlimb mouse model. In accordance with the in- 
vitro data, AS-IV-pretreated ADSCs significantly promoted the recovery of blood perfusion and capillary density of gastrocnemius muscle in ischemic hindlimbs. In tissue sections, the ADSC/AS-IV group also had less inflammatory infiltration and muscle degeneration. However, the specific mechanism of action may require further molecular biology experiments, and further investigation through clinical studies to test the long-term safety and effectiveness of this method.

\section{Conclusions}

In conclusion, we demonstrated that ADSCs pretreated with AS-IV exhibited better migration and angiogenesis capabilities to enhance the therapeutic function of stem cells, and can be used to treat lower limb ischemic diseases in vivo, possibly because AS-IV promotes the expression of CXCR2, which leads to FAK phosphorylation. Taken together, AS-IV pretreatment can be used as one of the methods to improve the therapeutic efficacy of ADSCs, and could bring new insights into the treatment of severe limb ischemia.

\section{Abbreviations}

\begin{tabular}{|ll|}
\hline ADSC & adipose stem cell \\
\hline CLI & severe limb ischemia \\
\hline MSC & mesenchymal stem cell \\
\hline AS-IV & Astragaloside IV \\
\hline HUVEC & Human umbilical vein endothelial cell \\
\hline FAK & focal adhesion kinase \\
\hline VEGF & vascular endothelial growth factor \\
\hline HIF-1a & hypoxia-inducible factor-1a \\
\hline PBS & phosphate buffered saline \\
\hline DMSO & dimethyl sulfoxide \\
\hline DMEM & Dulbecco's modified Eagle's medium \\
\hline DMEM/F-12 & Dulbecco's modified Eagle's medium F-12 \\
\hline MTT & 3-(4,5-dimethylthiazole-2-yl) -2,5-diphenyltetrazolium bromide \\
\hline CM & conditioned medium \\
\hline PVDF & polyvinylidene fluoride \\
\hline Matrigel & matrix gel \\
\hline PXN & paxillin \\
\hline
\end{tabular}




\section{Declarations}

\section{Acknowledgments}

We thank the Department of Pharmacy of the Air Force Medical University of China for their help with the laser Doppler instrument and appreciate our colleagues for their valuable efforts and comments on this paper.

\section{Authors' contributions}

W.Y. W, Z.K. S and Y.N. T performed the experiments. B.Y. C, J.X. C, and J.X. H analyzed and interpreted the data. S.Y. $L$ and W.Y. W wrote the manuscript. J.Y. L and M.Z. Z provided critical suggestions. S. L, Y.F. $M$ and L.W. $Z$ revised the manuscript critically for important intellectual content. S.Y. $L$ designed and supervised the project. All authors discussed the results and commented on the manuscript. The authors read and approved the final manuscript.

\section{Funding}

This work was supported by the National Natural Science Foundation of China (No: 81472747).

\section{Availability of data and materials}

The datasets generated/analyzed during the current study are available.

\section{Ethics approval and consent to participate}

This study was approved and supervised by the Ethical Committee of the First Affiliated Hospital of Medical College, Xi'an Jiaotong University, Xi'an, China. The study was conducted in accordance with the Declaration of Helsinki principles.

\section{Consent for publication}

The manuscript has been approved by all authors for publication.

\section{Conflict of interest}

The authors declare no conflict of interest. 


\section{References}

1. Aronow WS: Peripheral arterial disease of the lower extremities. Archives of medical science : AMS 2012, 8(2):375-388.

2. Mamidi MK, Pal R, Dey S, Bin Abdullah BJ, Zakaria Z, Rao MS, Das AK: Cell therapy in critical limb ischemia: current developments and future progress. Cytotherapy 2012, 14(8):902-916.

3. Tateishi-Yuyama E, Matsubara H, Murohara T, Ikeda U, Shintani S, Masaki H, Amano K, Kishimoto Y, Yoshimoto $\mathrm{K}$, Akashi $\mathrm{H}$ et al: Therapeutic angiogenesis for patients with limb ischaemia by autologous transplantation of bone-marrow cells: a pilot study and a randomised controlled trial. Lancet 2002, 360(9331):427-435.

4. Zuk PA, Zhu M, Ashjian P, De Ugarte DA, Huang JI, Mizuno H, Alfonso ZC, Fraser JK, Benhaim P, Hedrick MH: Human adipose tissue is a source of multipotent stem cells. Mol Biol Cell 2002, 13(12):4279-4295.

5. Potdar PD, Jethmalani YD: Human dental pulp stem cells: Applications in future regenerative medicine. World journal of stem cells 2015, 7(5):839-851.

6. Orciani M, Di Primio R: Skin-derived mesenchymal stem cells: isolation, culture, and characterization. Methods in molecular biology 2013, 989:275-283.

7. Li XL, Gan KX, Song GY, Wang C: VEGF gene transfected umbilical cord mesenchymal stem cells transplantation improve the lower limb vascular lesions of diabetic rats. J Diabetes Complicat 2015, 29(7):872-881.

8. Liew A, O'Brien $T$ : Therapeutic potential for mesenchymal stem cell transplantation in critical limb ischemia. Stem cell research \& therapy 2012, 3(4):28.

9. Lu HY, Wang F, Mei H, Wang SQ, Cheng LM: Human Adipose Mesenchymal Stem Cells Show More Efficient Angiogenesis Promotion on Endothelial Colony-Forming Cells than Umbilical Cord and Endometrium. Stem Cells Int 2018, 2018.

10. Hutchings G, Janowicz K, Moncrieff L, Dompe C, Strauss E, Kocherova I, Nawrocki MJ, Kruszyna L, Wasiatycz G, Antosik P et al: The Proliferation and Differentiation of Adipose-Derived Stem Cells in Neovascularization and Angiogenesis. International journal of molecular sciences 2020, 21(11).

11. Min Y, Han S, Aae Ryu H, Kim SW: Human adipose mesenchymal stem cells overexpressing dual chemotactic gene showed enhanced angiogenic capacity in ischaemic hindlimb model. Cardiovascular research 2018, 114(10):1400-1409.

12. Cheng S, Zhang X, Feng Q, Chen J, Shen L, Yu P, Yang L, Chen D, Zhang H, Sun W et al: Astragaloside IV exerts angiogenesis and cardioprotection after myocardial infarction via regulating PTEN/PI3K/Akt signaling pathway. Life sciences 2019, 227:82-93.

13. Feng B, Zhang Q, Wang X, Sun X, Mu X, Dong H: Effect of Andrographolide on Gene Expression Profile and Intracellular Calcium in Primary Rat Myocardium Microvascular Endothelial Cells. Journal of cardiovascular pharmacology 2017, 70(6):369-381. 
14. Jiang B, Yang YJ, Dang WZ, Li H, Feng GZ, Yu XC, Shen XY, Hu XG: Astragaloside IV reverses simvastatin-induced skeletal muscle injury by activating the AMPK-PGC-1alpha signalling pathway. Phytotherapy research : PTR 2020, 34(5):1175-1184.

15. Wang SG, $\mathrm{Xu} Y$, Chen JD, Yang $\mathrm{CH}$, Chen $\mathrm{XH}$ : Astragaloside IV stimulates angiogenesis and increases nitric oxide accumulation via JAK2/STAT3 and ERK1/2 pathway. Molecules 2013, 18(10):1280912819.

16. Xu F, Cui WQ, Wei Y, Cui J, Qiu J, Hu LL, Gong WY, Dong JC, Liu BJ: Astragaloside IV inhibits lung cancer progression and metastasis by modulating macrophage polarization through AMPK signaling. Journal of experimental \& clinical cancer research : CR 2018, 37(1):207.

17. Zhang L, Liu Q, Lu L, Zhao X, Gao X, Wang Y: Astragaloside IV stimulates angiogenesis and increases hypoxia-inducible factor-1alpha accumulation via phosphatidylinositol 3-kinase/Akt pathway. The Journal of pharmacology and experimental therapeutics 2011, 338(2):485-491.

18. Fang J, Ding M, Yang L, Liu LZ, Jiang BH: PI3K/PTEN/AKT signaling regulates prostate tumor angiogenesis. Cellular signalling 2007, 19(12):2487-2497.

19. Limbourg A, Korff T, Napp LC, Schaper W, Drexler H, Limbourg FP: Evaluation of postnatal arteriogenesis and angiogenesis in a mouse model of hind-limb ischemia. Nature protocols 2009, 4(12):1737-1746.

20. Ai M, Yan CF, Xia FC, Zhou SL, He J, Li CP: Safety and efficacy of cell-based therapy on critical limb ischemia: A meta-analysis. Cytotherapy 2016, 18(6):712-724.

21. Jaluvka F, Ihnat P, Madaric J, Vrtkova A, Janosek J, Prochazka V: Current Status of Cell-Based Therapy in Patients with Critical Limb Ischemia. International journal of molecular sciences 2020, 21(23).

22. Eggenhofer E, Benseler V, Kroemer A, Popp FC, Geissler EK, Schlitt HJ, Baan CC, Dahlke MH, Hoogduijn MJ: Mesenchymal stem cells are short-lived and do not migrate beyond the lungs after intravenous infusion. Frontiers in immunology 2012, 3:297.

23. Ma X, Zhang K, Li H, Han S, Ma Z, Tu P: Extracts from Astragalus membranaceus limit myocardial cell death and improve cardiac function in a rat model of myocardial ischemia. Journal of ethnopharmacology 2013, 149(3):720-728.

24. Nitzsche F, Muller C, Lukomska B, Jolkkonen J, Deten A, Boltze J: Concise Review: MSC Adhesion Cascade-Insights into Homing and Transendothelial Migration. Stem Cells 2017, 35(6):1446-1460.

25. Lamalice L, Le Boeuf F, Huot J: Endothelial cell migration during angiogenesis. Circulation research 2007, 100(6):782-794.

26. Shireman PK: The chemokine system in arteriogenesis and hind limb ischemia. Journal of vascular surgery 2007, 45 Suppl A:A48-56.

27. Li MW, Sun XF, Ma L, Jin L, Zhang WF, Xiao M, Yu Q: SDF-1/CXCR4 axis induces human dental pulp stem cell migration through FAK/PI3K/Akt and GSK3 beta/beta-catenin pathways. Sci Rep-Uk 2017, 7. 
28. Yang G, Rosen DG, Liu GZ, Yang F, Guo XQ, Xiao X, Xue FX, Mercado-Uribe I, Huang JT, Lin SH et al: CXCR2 Promotes Ovarian Cancer Growth through Dysregulated Cell Cycle, Diminished Apoptosis, and Enhanced Angiogenesis. Clin Cancer Res 2010, 16(15):3875-3886.

29. Barnawi R, Al-Khaldi S, Bakheet T, Fallatah M, Alaiya A, Ghebeh H, Al-Alwan M: Fascin Activates betaCatenin Signaling and Promotes Breast Cancer Stem Cell Function Mainly Through Focal Adhesion Kinase (FAK): Relation With Disease Progression. Front Oncol 2020, 10.

30. Zhao X, Guan JL: Focal adhesion kinase and its signaling pathways in cell migration and angiogenesis. Adv Drug Deliver Rev 2011, 63(8):610-615.

31. Peng X, Ueda H, Zhou HM, Stokol T, Shen TL, Alcaraz A, Nagy T, Vassalli JD, Guan JL: Overexpression of focal adhesion kinase in vascular endothelial cells promotes angiogenesis in transgenic mice. Cardiovascular research 2004, 64(3):421-430.

32. Meng FB, Rui YF, Xu LL, Wan C, Jiang XH, Li G: Aqp1 Enhances Migration of Bone Marrow Mesenchymal Stem Cells Through Regulation of FAK and beta-Catenin. Stem Cells Dev 2014, 23(1):66-75.

\section{Figures}


A

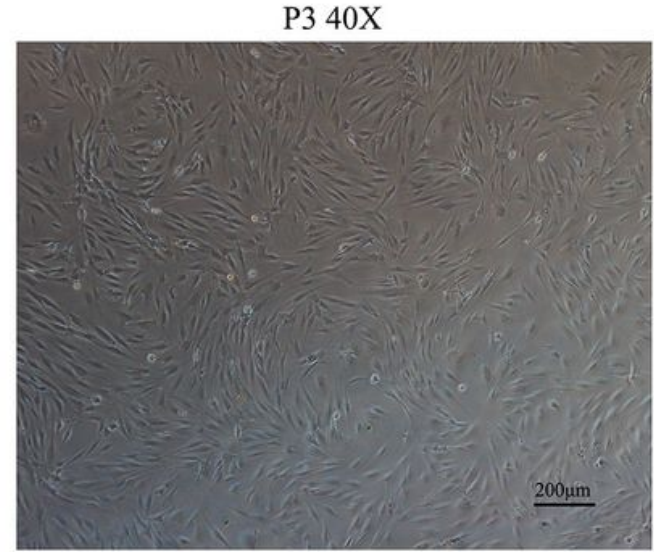

B
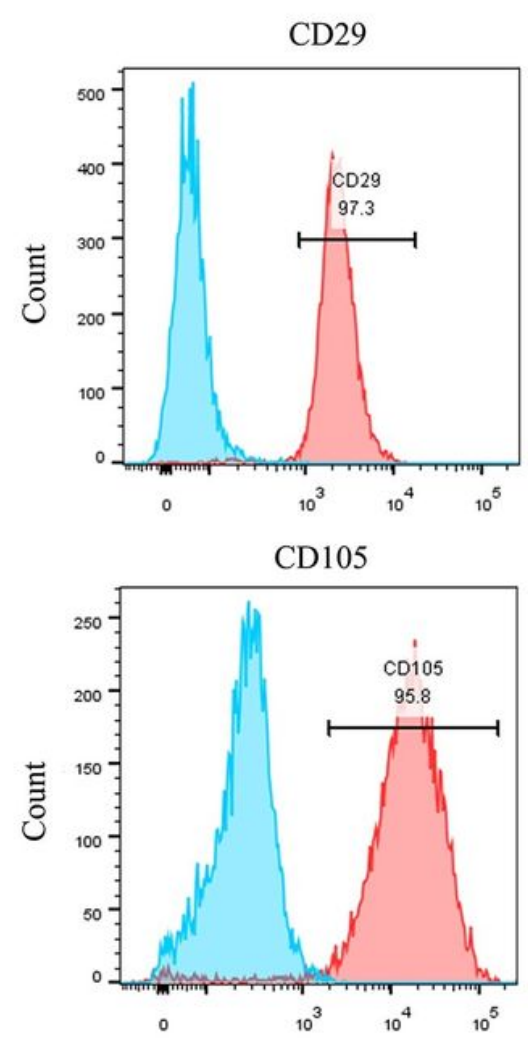

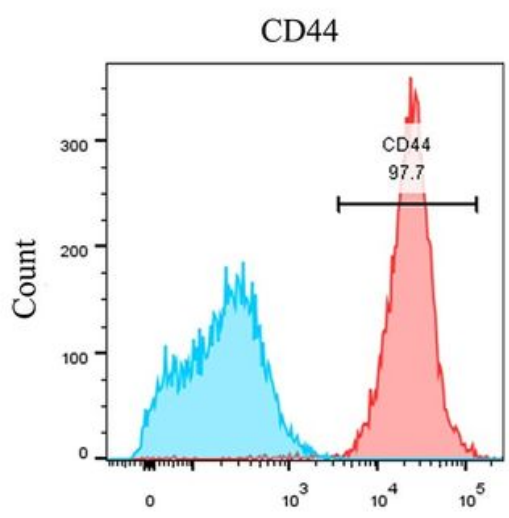

P3 100X
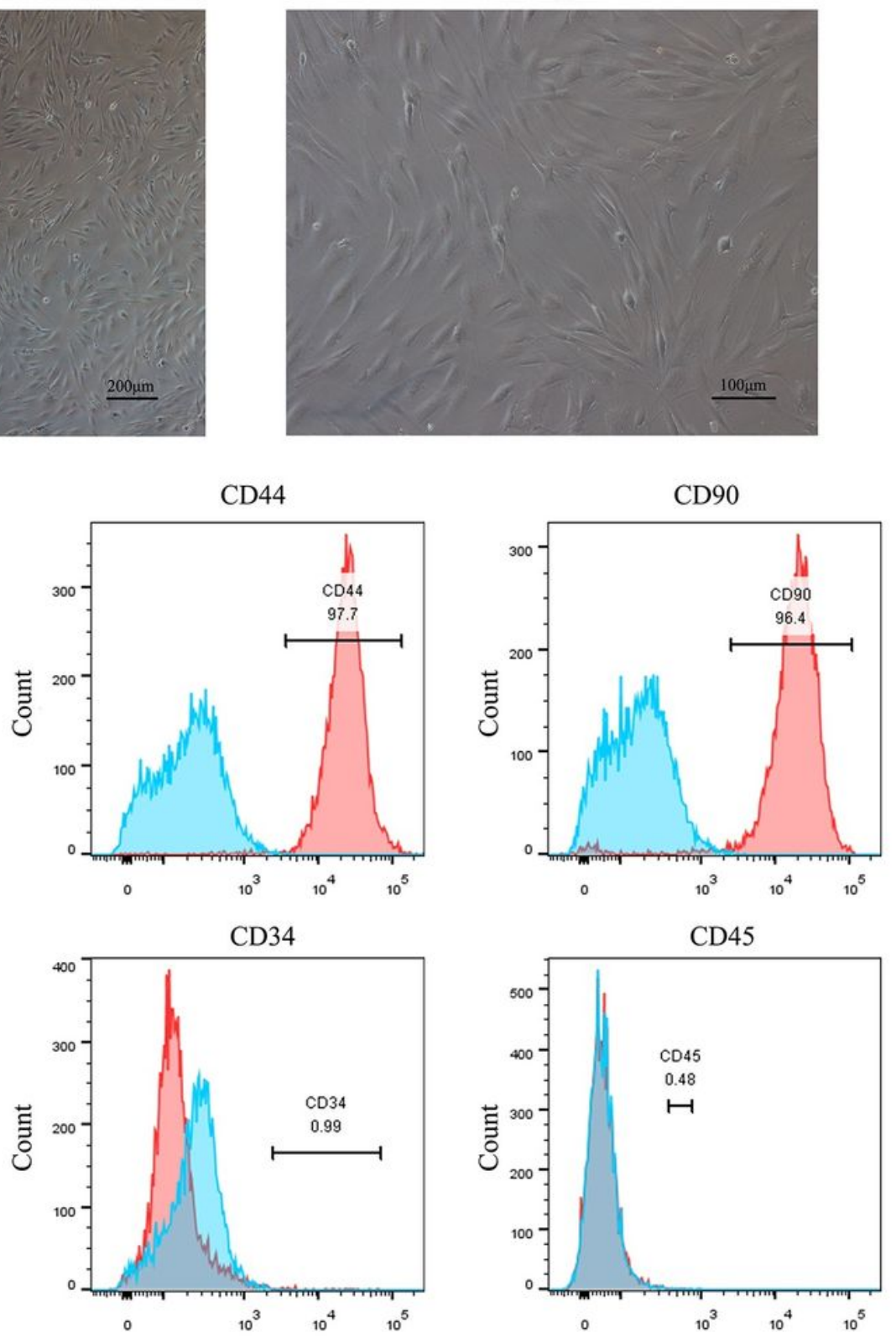

Figure 1

Characterization and flow cytometry analysis of ADSCs. (A) P3 ADSCs under 40x optical lenses (left) and $100 \times$ optical lenses (right). Cells showed a fibroblast-like shape and were distributed in a whirlpool shape. (B) Flow cytometry analysis of the ADSCs surface markers CD29, CD44, CD90 and CD105. Hematopoietic cell-specific surface antigen CD34 and leukocyte surface marker CD45 were also analyzed. Blue peaks represent matched negative controls. 
A

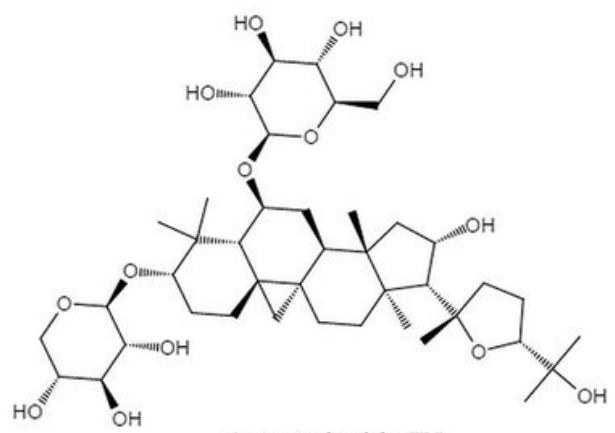

Astragaloside IV
B

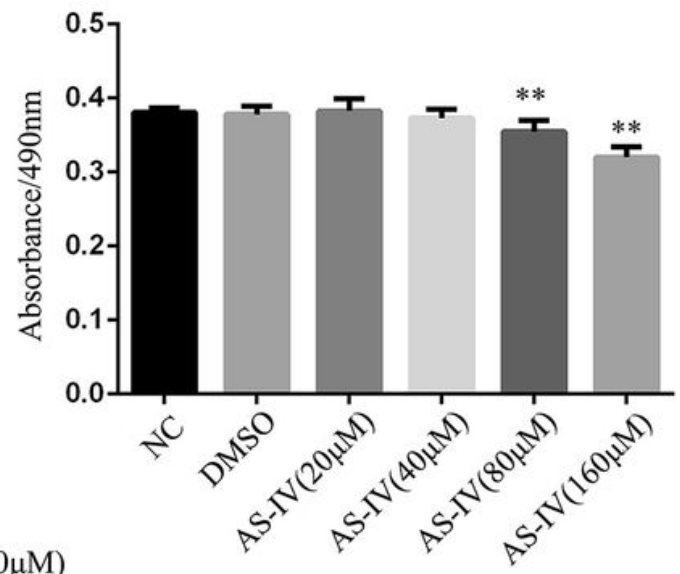

C NC DMSO $\operatorname{AS}-\operatorname{IV}(20 \mu \mathrm{M})$ AS-IV $(40 \mu \mathrm{M})$
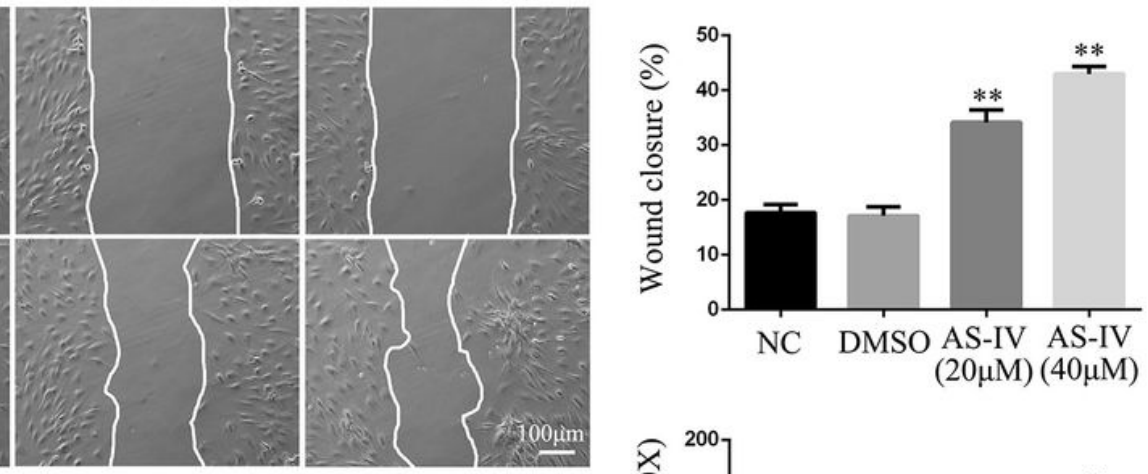

D
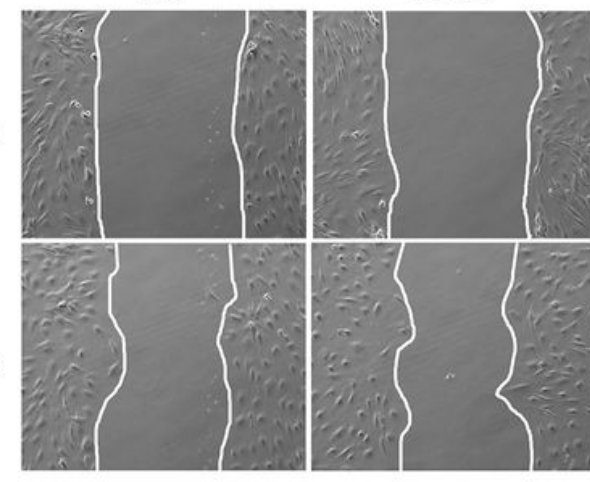

AS-IV $(20 \mu \mathrm{M})$

AS-IV $(40 \mu \mathrm{M})$
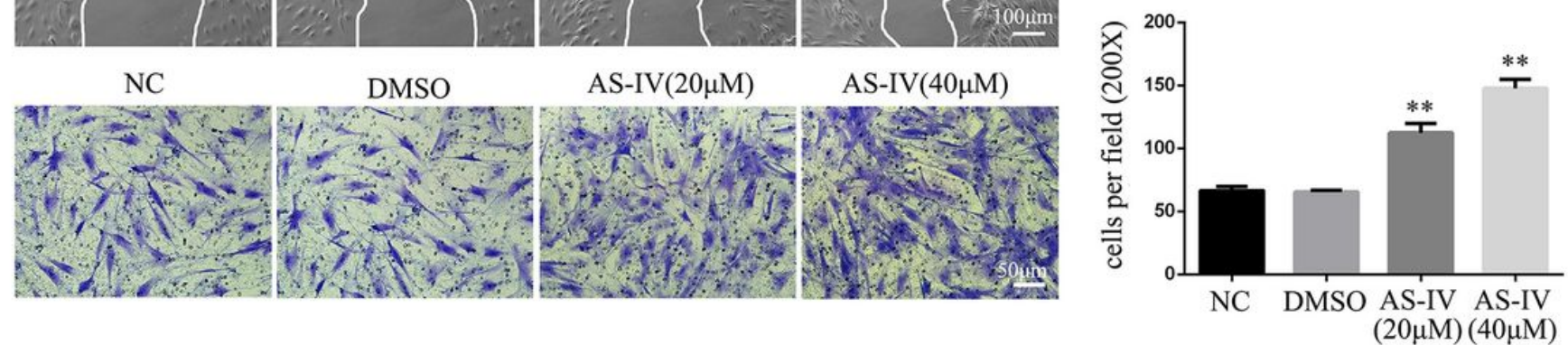

\section{Figure 2}

Effects of AS-IV on ADSC viability and migration. (A) The chemical structure of AS-IV. (B) ADSC growth after treatment with AS-IV for $48 \mathrm{~h}$ was assessed by MTT. The migration ability of ADSCs after treatment with AS-IV at different concentrations for $48 \mathrm{~h}$ was assessed by wound healing assay (C) and Transwell assay (D), and the quantitative results are shown on the right side, ${ }^{\star \star} p<0.01$ versus the NC group.

\section{Figure 3}

Effects of AS-IV-treated ADSC-conditioned medium on HUVEC recruitment and Matrigel angiogenesis. (A) Photographs of HUVEC scratch closure after culture with CM from ADSCs treated with different concentrations of AS-IV. The quantitative results are shown on the right side. (B) Photographs of the Transwell experiment illustrate HUVEC recruitment of ADSC CM after treatment with different concentrations of AS-IV. Quantitative results of cell migration are shown on the right side. (C) Representative images of Matrigel tube formation potential from different CMs. The histograms below count the number of tubes and total length of different groups. ${ }^{*} p<0.05$ and ${ }^{*} p<0.01$ versus NC group. 
A

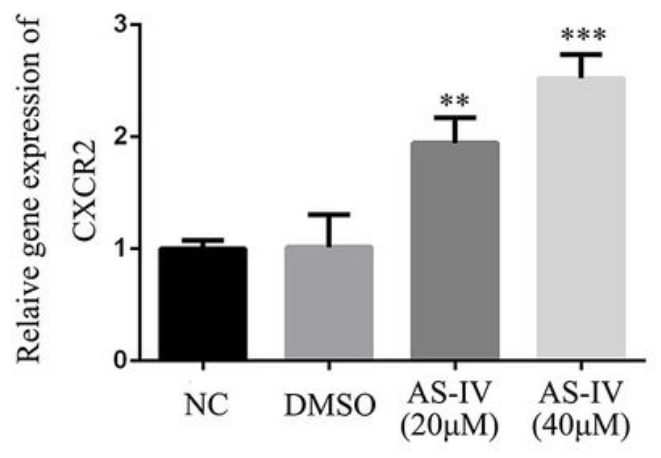

$\mathrm{C}$

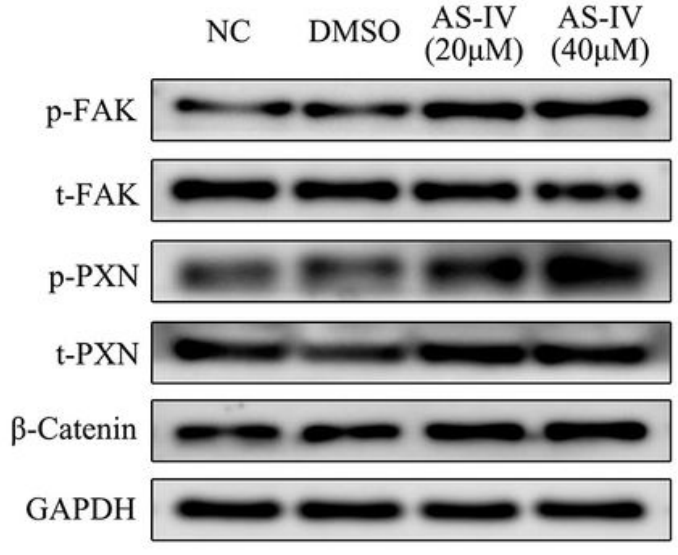

$\mathrm{D}$

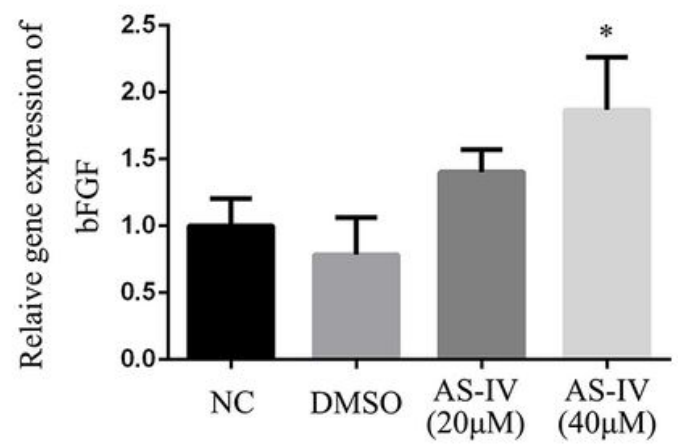

B
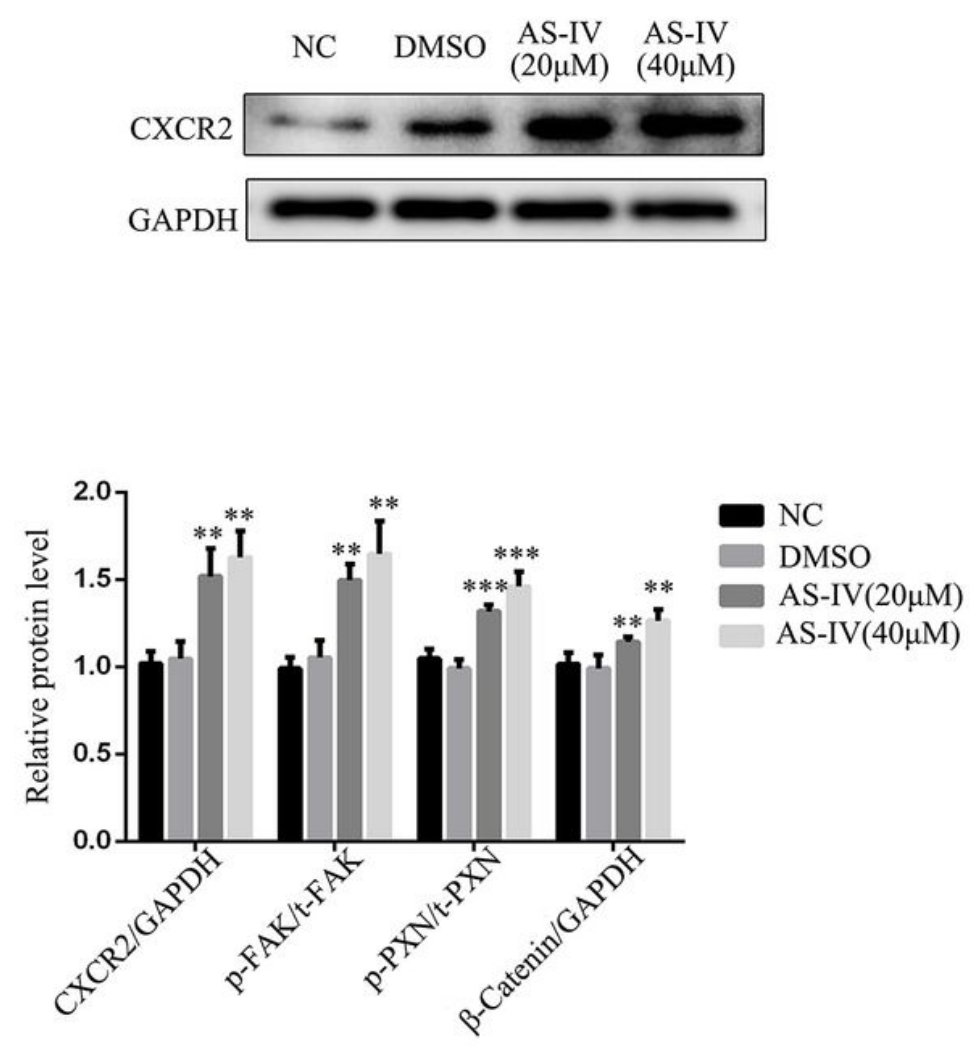

E

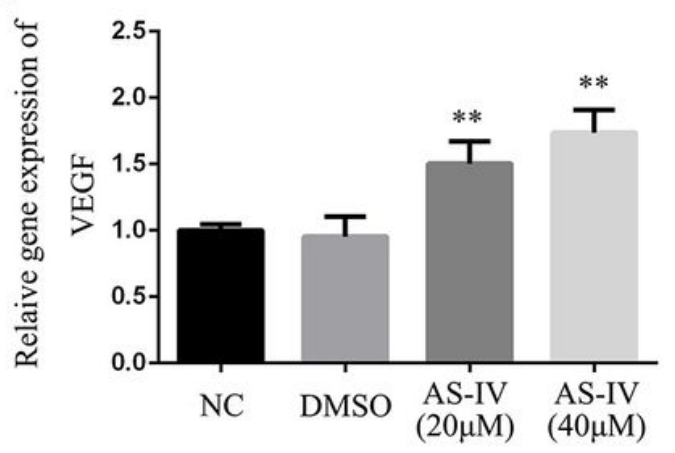

\section{Figure 4}

AS-IV mediated concentration-dependent effects on CXCR2, p-FAK, p-PXN and $\beta$-Catenin protein expression and mRNA expression of angiogenesis-related factors. (A) Quantitative RT-PCR analysis of CXCR2 mRNA levels in ADSCs and ADSCs pretreated with AS-IV or DMSO. (B) Western blot analysis of CXCR2 expression in each group. GAPDH was used as a control. (C) Western blot analysis of p-FAK, pPXN and $\beta$-Catenin expression in each group. GAPDH was used as a control, and quantitative results of the relative protein level are shown on the right side. (D) Quantitative RT-PCR analysis of the mRNA levels of the angiogenesis-related factors bFGF and VEGF in each group. ${ }^{*} p<0.05$ and ${ }^{* *} p<0.01$ and ${ }^{* * *} \mathrm{p}<0.001$ versus the NC group. 


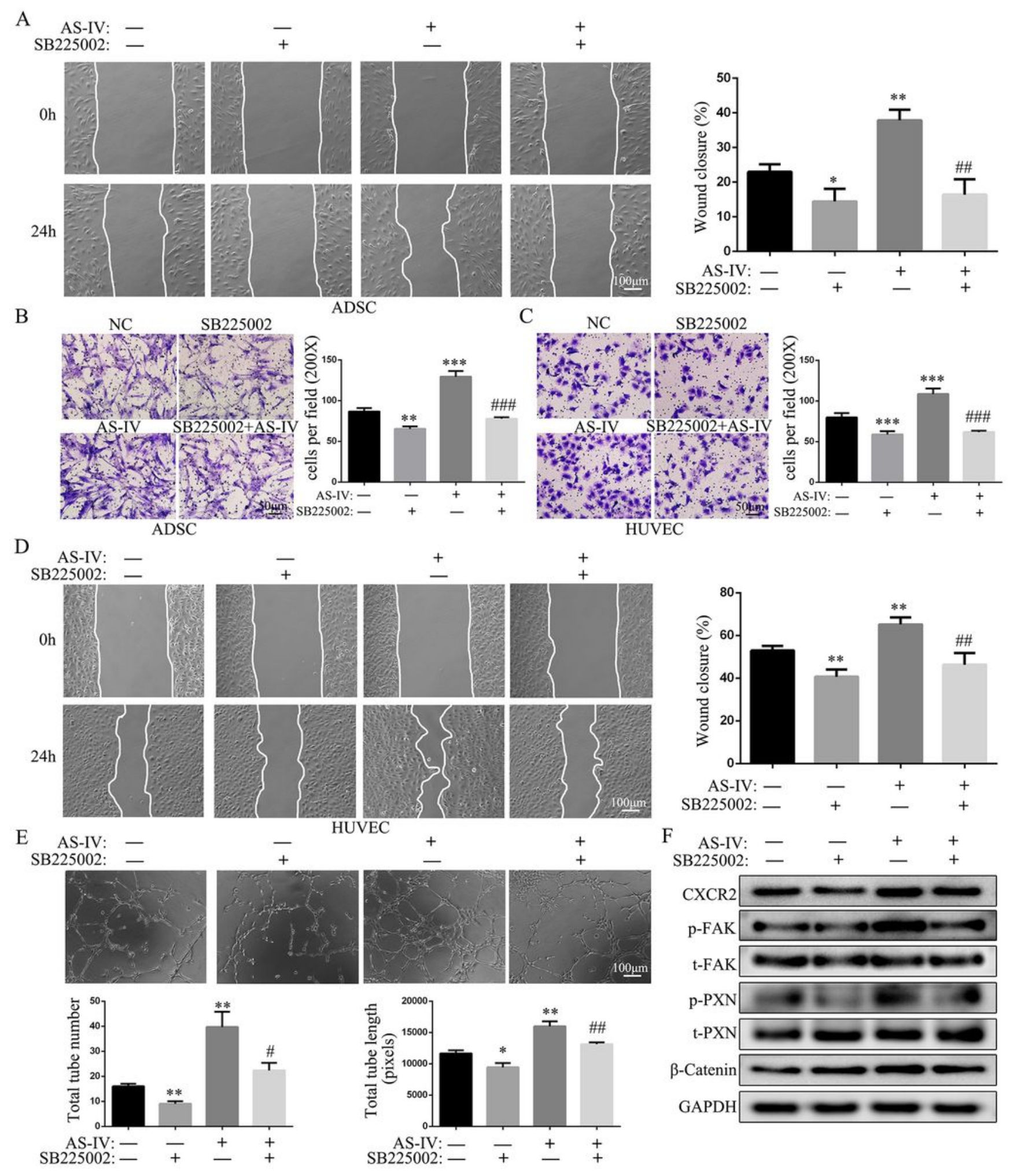

\section{Figure 5}

SB225002 inhibited the increase in migration and angiogenesis of ADSCs induced by CXCR2 overexpression. (A) After preincubation with SB225002 $(1 \mu \mathrm{M})$ for 1 hour, ADSCs were treated with AS-IV $(40 \mu \mathrm{M})$ for $48 \mathrm{~h}$, and a wound healing assay was used to evaluate the healing ability. Quantitative results are shown on the right side. (B)Transwell assay to evaluate the migration ability of ADSCs treated with AS-IV after preincubation with SB225002 or not, and quantify the number of cells passed. (C) Transwell 
assay to evaluate the recruitment effect on HUVECs in ADSC CM. ADSCs were pretreated with AS-IV and SB225002, and quantitative results are shown on the right side. (D) Wound healing assay to evaluate the healing ability of HUVECs treated with each ADSC CM. Quantitative of wound closure is shown on the right side. (E) Representative images of Matrigel tube formation potential from different CMs. The histograms below count the number of tubes and total length of different groups. ( $F$ ) After preincubation with SB225002 or AS-IV, ADSCs were subjected to Western blots and detected with the indicated antibodies. ${ }^{*} p<0.05,{ }^{*} p<0.01$ and ${ }^{* \star *} p<0.001$ versus the control group. $\# p<0.05, \# \# p<0.01$ and $\# \# \# p<0.001$ versus the AS-IV group. 


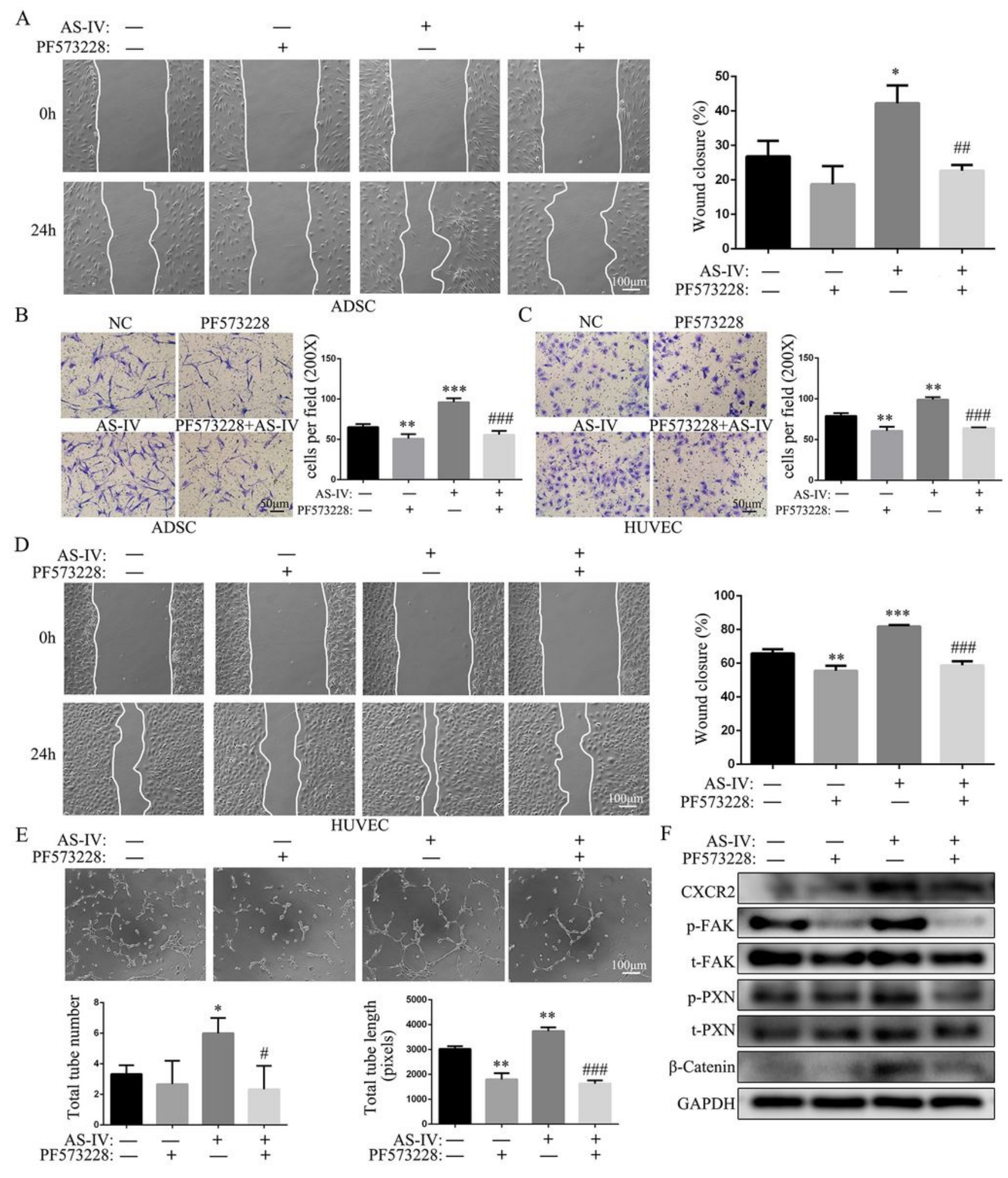

\section{Figure 6}

Effect of the FAK phosphorylation inhibitor PF573228 on ADSC migration and angiogenesis ability. (A) After preincubation with PF573228 $(10 \mu \mathrm{M})$ for 1 hour, ADSCs were treated with AS-IV $(40 \mu \mathrm{M})$ for $48 \mathrm{~h}$ and a wound healing assay was used to evaluate the healing ability. Quantitative results are shown on the right side. (B)Transwell assay to evaluate the migration ability of ADSCs treated with AS-IV after preincubation with SB225002 or not and quantify the number of cells passed. (C) Transwell assay to 
evaluate the recruitment effect on HUVECs in ADSC CM. ADSCs were pretreated with AS-IV and PF573228, and quantitative results are shown on the right side. (D) Wound healing assay to evaluate the healing ability of HUVECs treated with each ADSC CM. Quantitative wound closure is shown on the right side. (E) Representative images of Matrigel tube formation potential from different ADSC CMs. The histograms below count the number of tubes and total length of different groups. (F) After preincubation with PF573228 or AS-IV, ADSCs were subjected to Western blots and detected with the indicated antibodies. ${ }^{*} p<0.05,{ }^{* *} p<0.01$ and ${ }^{* * *} p<0.001$ versus the control group. $\# p<0.05, \# \# p<0.01$ and \#\#\#p<0.001 versus the AS-IV group.

A

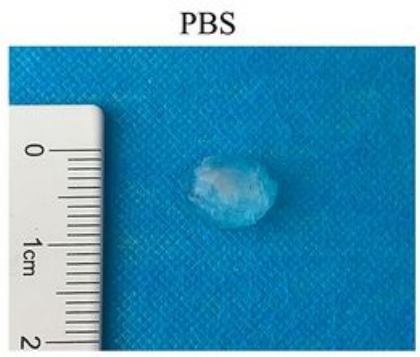

$\mathrm{C}$

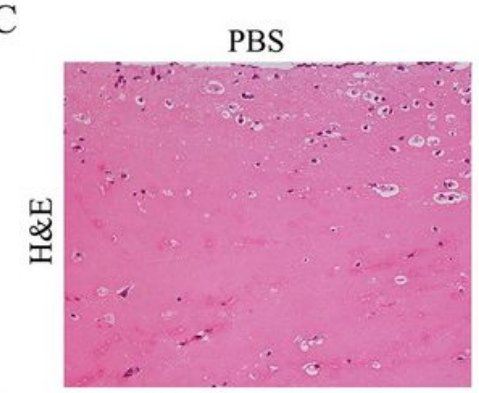

$\mathrm{D}$
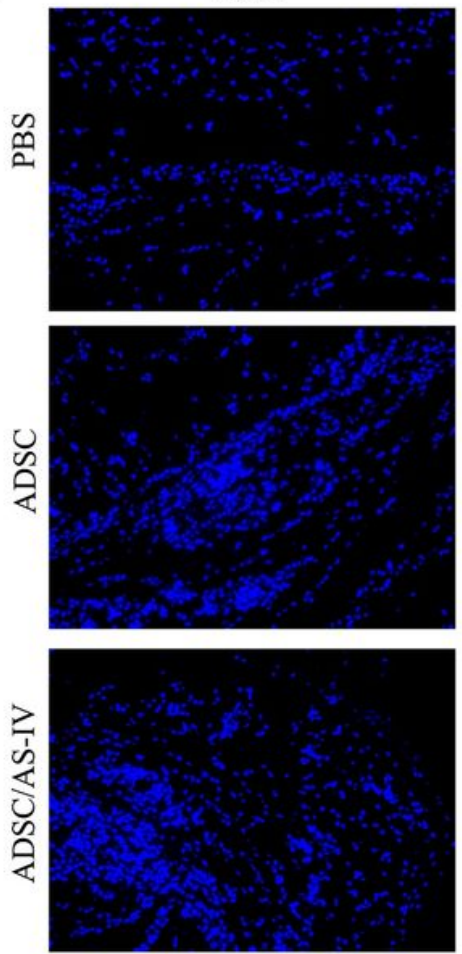

ADSC

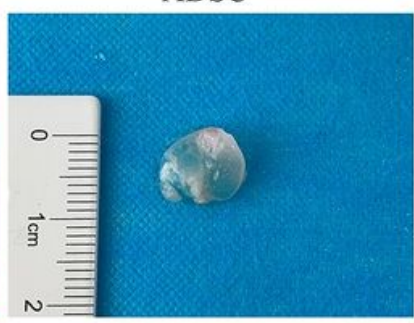

ADSC

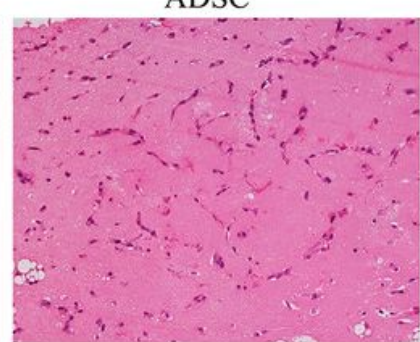

$\mathrm{CD} 31$
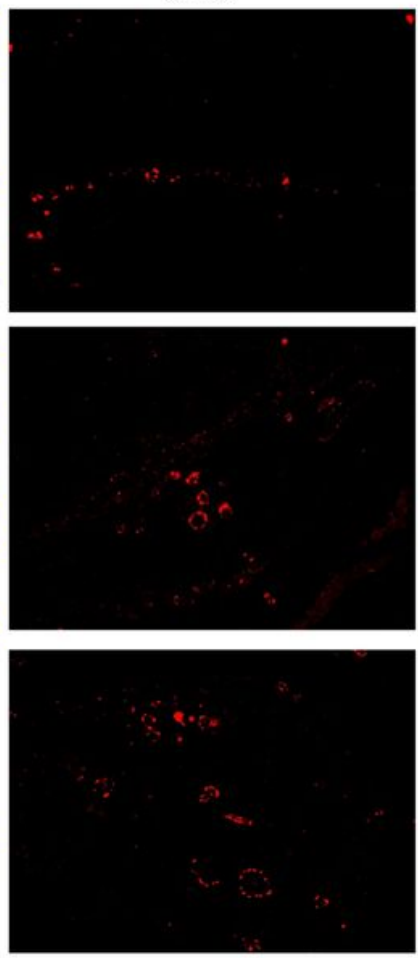

ADSC/AS-IV
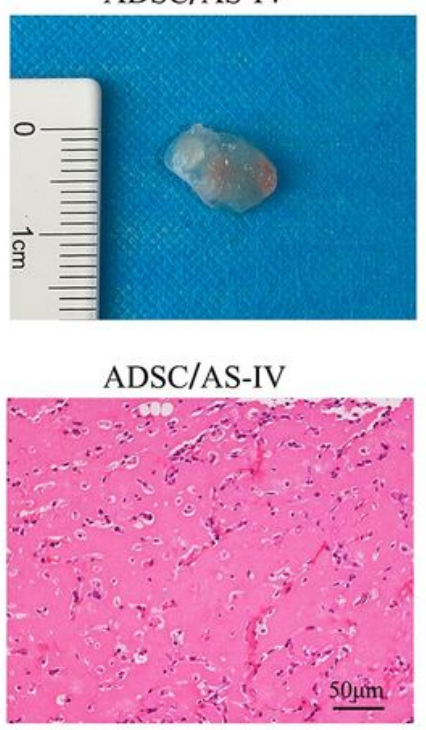

Merged
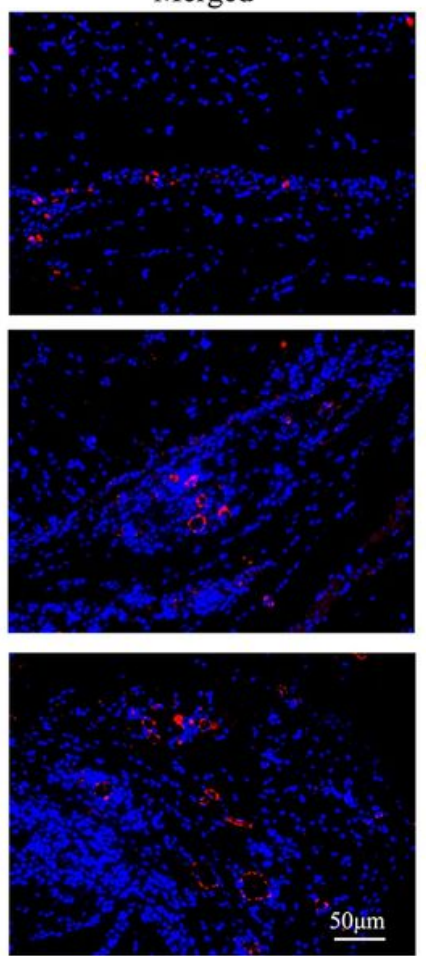

B

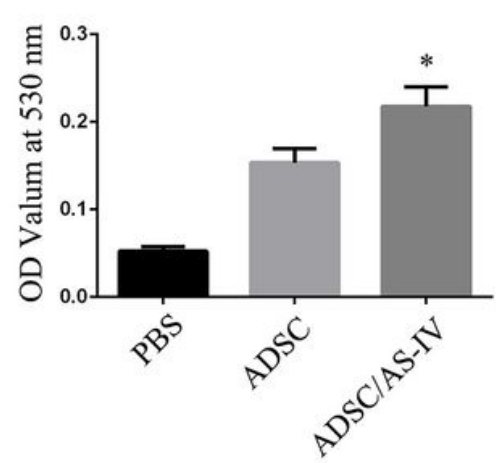

E

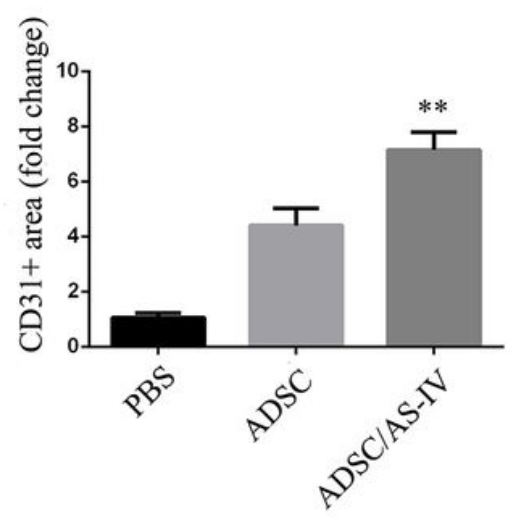

Figure 7 
Vasculogenic potential in vivo measured by Matrigel plug assay. (A) Representative photographs of Matrigel plugs injected with PBS, ADSCs or ADSCs/AS-IV at Day 14. (B) OD value of hemoglobin concentration in Matrigel plug. (C) H\&E-stained sections of Matrigel plug. (D) Immunofluorescent staining of CD31 in sections of Matrigel plugs. Quantification of the CD31+ area was performed (E). ${ }^{*} p<0.05$ and $* * p<0.01$ versus ADSC group.

A
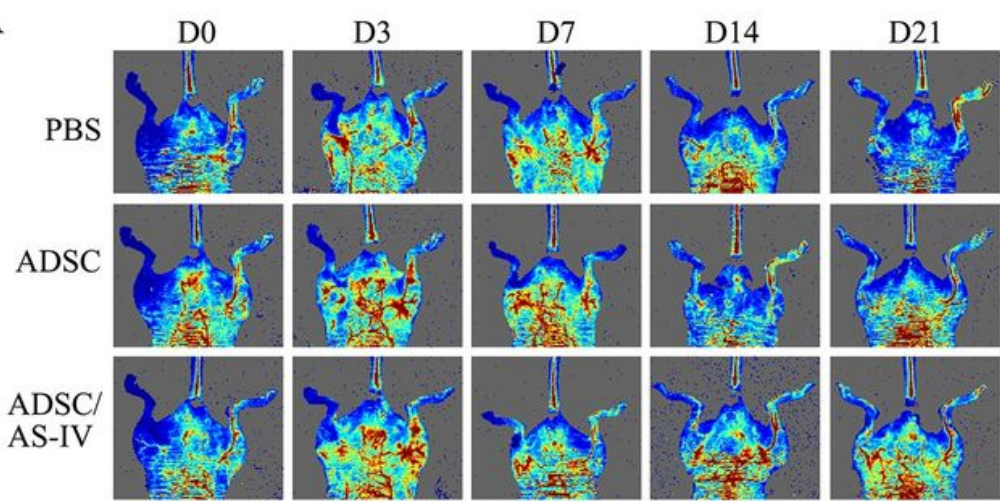

B
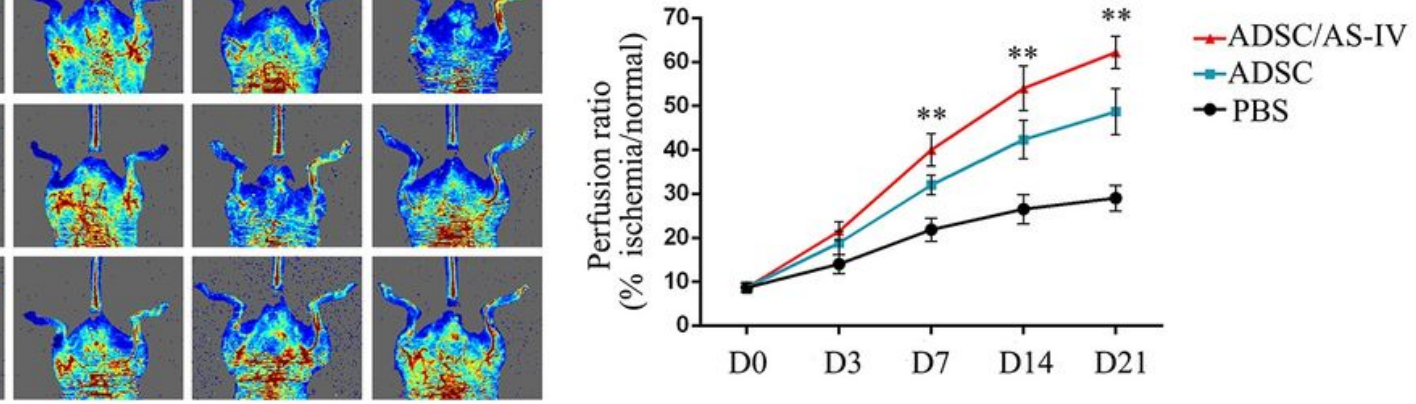

$\mathrm{C}$

DAPI

CD31
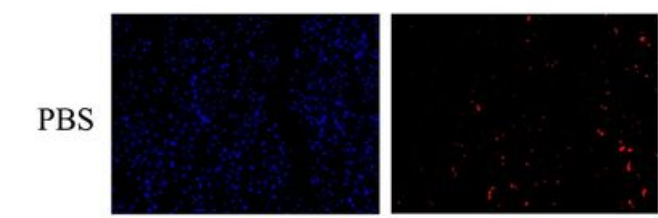

Merged

$\mathrm{D}$
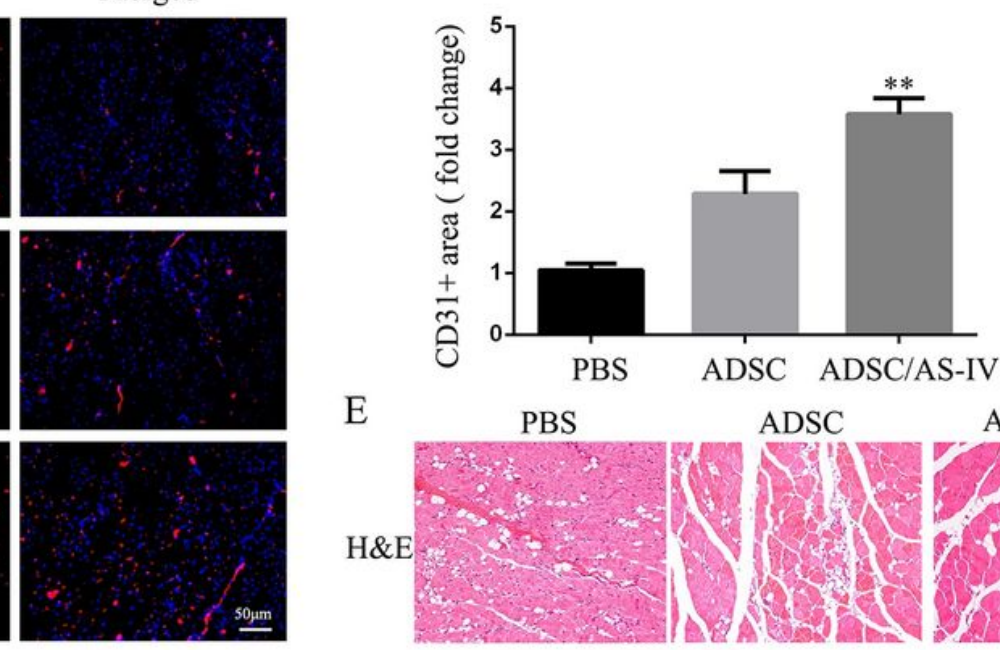

E

PBS

ADSC

ADSC/AS-IV
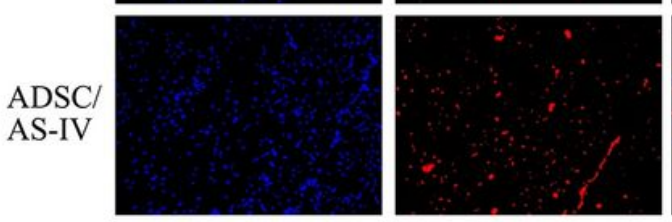

H\&E

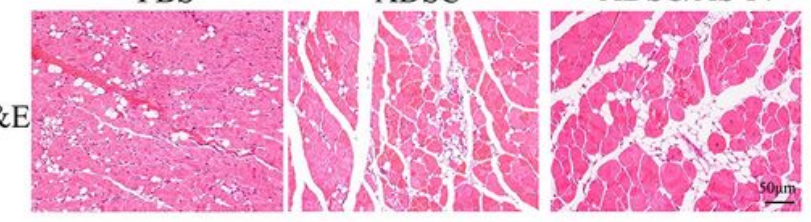

\section{Figure 8}

Recovery of blood flow and therapeutic mechanism in a mouse model of hind limb ischemia after cell transplantation. (A) Representative laser Doppler perfusion images of the recovery of blood flow in ischemic hind limbs 21 days after intramuscular injection with PBS, ADSCs or ADSCs/AS-IV. (B) Blood perfusion ratio between different groups at 21 days. (C) Immunofluorescent staining for CD31 in sections of gastrocnemius tissue sections at Day 21. (D) The proportion of CD31+ area in each group. (E) H\&Estained sections of gastrocnemius tissue sections at Day 14 . ${ }^{*} \mathrm{p}<0.01$ versus ADSC group.

\section{Supplementary Files}

This is a list of supplementary files associated with this preprint. Click to download.

- SupplementalFig.1.tif 